\title{
Phase diagram in the imaginary chemical potential region and extended $\mathbb{Z}_{3}$ symmetry
}

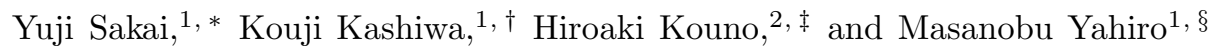 \\ ${ }^{1}$ Department of Physics, Graduate School of Sciences, Kyushu University, Fukuoka 812-8581, Japan \\ ${ }^{2}$ Department of Physics, Saga University, Saga 840-8502, Japan
}

(Dated: October 27, 2018)

\begin{abstract}
Phase transitions in the imaginary chemical potential region are studied by the Polyakov loop extended Nambu-Jona-Lasinio (PNJL) model that possesses the extended $\mathbb{Z}_{3}$ symmetry. The extended- $\mathbb{Z}_{3}$ invariant quantities such as the partition function, the chiral condensate and the modified Polyakov loop have the Roberge-Weiss (RW) periodicity. There appear four types of phase transitions; deconfinement, chiral, Polyakov-loop RW and chiral RW transitions. The orders of the chiral and deconfinement transitions depend on the presence or absence of current quark mass, but those of the Polyakov-loop RW and chiral RW transitions do not. The scalar-type eight-quark interaction newly added in the model makes the chiral transition line shift to the vicinity of the deconfinement transition line.
\end{abstract}

PACS numbers: 11.30.Rd, 12.40.-y

\section{INTRODUCTION}

The lattice QCD (LQCD) simulations have become feasible for thermal systems at zero quark chemical potential $(\mu)$ [1]. As for $\mu^{2}>0$, however, lattice QCD has the well-known sign problem, and the results are still far from perfection; for example, see Ref. [2] and references therein.

Several approaches have been proposed to solve the sign problem. One of them is the use of imaginary chemical potential, since the fermionic determinant appearing in the Euclidean partition function is real in the case; for example, see Refs. [3, 4, [5, 6, 7] and references therein. If the physical quantity such as chiral condensate is known in the imaginary $\mu$ region, one can extrapolate it to the real $\mu$ region, until there appears a discontinuity. Furthermore, in principle, one can evaluate with the Fourier transformation the canonical partition function with fixed quark number from the grand canonical partition function with imaginary $\mu[8,9,10]$.

Roberge and Weiss (RW) [11] found that the partition function of $\mathrm{SU}(N)$ gauge theory with imaginary chemical potential $\mu=i \theta / \beta$ for fermion number

$$
\begin{aligned}
& Z(\theta)=\int D \psi D \bar{\psi} D A_{\mu} \exp \left[-\int d^{4} x\right. \\
& \left.\left\{\bar{\psi}\left(\gamma D-m_{0}\right) \psi-\frac{1}{4} F_{\mu \nu}^{2}-i \frac{\theta}{\beta} \bar{\psi} \gamma_{4} \psi\right\}\right],
\end{aligned}
$$

is a periodic function of $\theta$ with a period $2 \pi / N$, that is $Z(\theta+2 \pi k / N)=Z(\theta)$ for any integer $k$, by showing that $Z(\theta+2 \pi k / N)$ is reduced to $Z(\theta)$ with the $\mathbb{Z}_{N}$ transfor-

\footnotetext{
* sakai2scp@mbox.nc.kyushu-u.ac.jp

† kashiwa@phys.kyushu-u.ac.jp

tkounoh@cc.saga-u.ac.jp

$\S$ yahiro@phys.kyushu-u.ac.jp
}

mation

$$
\psi \rightarrow U \psi, \quad A_{\nu} \rightarrow U A_{\nu} U^{-1}-\frac{i}{g}\left(\partial_{\nu} U\right) U^{-1}
$$

where $U(x, \tau)$ are elements of $\operatorname{SU}(N)$ with $U(x, \beta)=$ $\exp (-2 i \pi k / N) U(x, 0)$. Here $\psi$ is the fermion field, $F_{\mu \nu}$ is the strength tensor of the gauge field $A_{\nu}$, and $\beta$ is the inverse of temperature $T$. The RW periodicity means that $Z(\theta)$ is invariant under the extended $\mathbb{Z}_{N}$ transformation

$\theta \rightarrow \theta+\frac{2 \pi k}{N}, \psi \rightarrow U \psi, A_{\nu} \rightarrow U A_{\nu} U^{-1}-\frac{i}{g}\left(\partial_{\nu} U\right) U^{-1}$

Quantities invariant under the extended $\mathbb{Z}_{N}$ transformation, such as the effective potential $\Omega(\theta)$ and the chiral condensate, keep the RW periodicity. Meanwhile, the Polyakov loop $\Phi$ is not invariant under the transformation (3), since it is transformed as $\Phi \rightarrow \Phi e^{-i 2 \pi k / N}$. In general, non-invariant quantities such as $\Phi$ do not have the periodicity. This problem can be solved by introducing the modified Polyakov loop $\Psi(\theta) \equiv \Phi \exp (i \theta)$ invariant under (3), as shown later.

Roberge and Weiss also showed with perturbation that in the high $T$ region $d \Omega(\theta) / d \theta$ and $\Phi(\theta)$ are discontinuous at $\theta=(2 k+1) \pi / N$, and also found with the strong coupled lattice theory that the discontinuity disappears in the low $T$ region. This is called the Polyakov-loop RW phase transition in this paper and it is observed in the lattice simulations [3, 4, [5, 6, 7].

Figure 1 shows a predicted phase diagram in the $\theta-T$ plane. The solid lines represent the Polyakov-loop RW phase transitions, and the dot-dashed lines do the chiral phase transitions predicted by the lattice simulation, although the result of the simulation is not conclusive since the current quark mass taken is much heavier than the realistic one $5 \sim 10 \mathrm{MeV}$.

The Polyakov-loop RW transition is not an ordinary first-order deconfinement transition. Although both transitions are defined by discontinuities of $\Phi$, the latter is a jump of the absolute value $|\Phi|$ from almost zero 


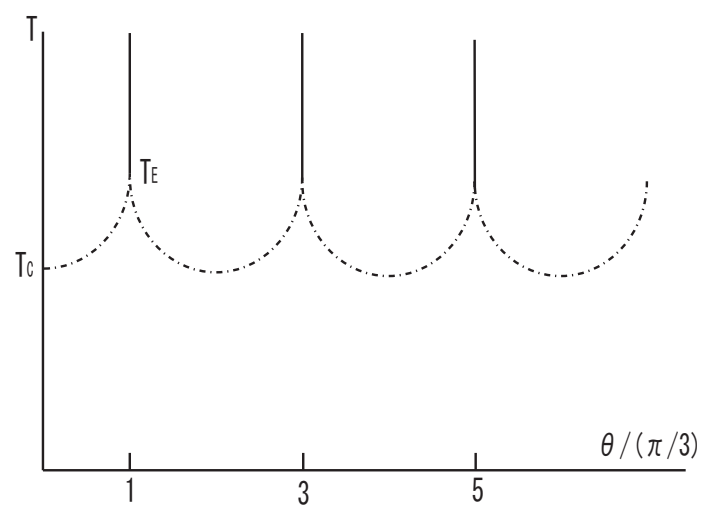

Fig. 1: The RW prediction on the QCD phase diagram in the $\theta-T$ plane. The solid lines represent the Polyakov-loop RW phase transitions, and the dot-dashed ones correspond to the chiral phase transitions.

to a finite value, but the former is a discontinuity of $\Phi$ in its phase, as shown later. The discontinuity means that the Polyakov-loop RW transition is surely first-order in the phase.

As shown later, the chiral condensate $\sigma$ is also not smooth on the Polyakov-loop RW transition lines (the solid lines of Fig. 1) unless $\sigma$ is zero. This is called the chiral RW transition in this paper. This is also not an ordinary second-order chiral transition. The former is a jump of $d \sigma / d \theta$ from a finite value to its minus sign, while the latter is a divergence of $d \sigma / d \theta$.

As an approach complementary to first-principle lattice simulations, one can consider several effective models. One of them is the Nambu-Jona-Lasinio (NJL) model [12]. Although the NJL model is a useful method for understanding the chiral symmetry breaking, this model does not possess a confinement mechanism. As a reliable model that can treat both the chiral and the deconfinement phase transition, we can consider the Polyakov loop extended NJL (PNJL) model $[13,14,15,16,17,18,19,20,21,22,23,24,25,26$. in which the deconfinement phase transition is described by the Polyakov loop.

The PNJL model has already been applied to the real chemical potential region and many interesting results, in particular on the relation between the chiral and the deconfinement phase transition, are reported [13, 14, 15, 16, 17, 18, 19, 20, 21, 22, 23, 24, 25, 26]. For example, the confinement mechanism shifts the critical endpoint of the chiral phase transition [27, 28, 29] to higher $T$ and lower $\mu[21,25]$. In order to confirm that these analyses are reliable, we should test the validity of the PNJL model by comparing the model results with the lattice ones. This is possible in the imaginary chemical potential region where the lattice simulation is feasible. If the PNJL model is successful in reproducing lattice results in the imaginary chemical potential region, this will imply not only that the PNJL model is reliable for both the real and imaginary chemical potential regions but also that the lattice results can be reasonably extrapolated to the real chemical potential region by using the PNJL model.

In the previous paper [30], we first applied the PNJL model to the imaginary chemical potential region and investigated the phase diagram in the chiral limit. Among many effective models, the PNJL model has both the chiral symmetry and the extended $\mathbb{Z}_{3}$ symmetry needed to reproduce the RW periodicity. We showed with the extended $\mathbb{Z}_{3}$ symmetry that the Polyakov-loop RW transition is first-order in the phase of $\Psi$ and the chiral RW transition is second-order. We also showed that both the crossover deconfinement and the second-order chiral transition take place in the $\theta-T$ plane, as expected. In this paper, we make more extensive analyses on the four phase transitions by newly adding quark mass term and scalar-type eight-quark interaction to the PNJL Lagrangian and seeing their effects on the transitions. Throughout these analyses, we will find that results of the PNJL model are consistent with all the lattice results.

This paper is organized as follows. In $\S 2$, we describe the PNJL model. In $\S 3$, the extended $\mathbb{Z}_{3}$ symmetry, which plays a crucial role in our analyses, are introduced. In $\S 4$, numerical results are presented. Section 5 is devoted to summary.

\section{THE PNJL MODEL}

The model we consider is the following two-flavor PNJL Lagrangian:

$$
\begin{aligned}
\mathcal{L}=\bar{q} & \left(i \gamma_{\nu} D^{\nu}-m_{0}\right) q \\
& +G_{\mathrm{s}}\left[(\bar{q} q)^{2}+\left(\bar{q} i \gamma_{5} \vec{\tau} q\right)^{2}\right]-\mathcal{U}\left(\Phi[A], \Phi[A]^{*}, T\right),
\end{aligned}
$$

where $q$ denotes the two-flavor quark field, $m_{0}$ does the current quark mass, and $D^{\nu}=\partial^{\nu}-i A^{\nu}-i \mu \delta_{0}^{\nu}$. The field $A^{\nu}$ is defined as $A^{\nu}=\delta_{0}^{\nu} g A_{a}^{0} \frac{\lambda^{a}}{2}$ with the gauge field $A_{a}^{\nu}$, the Gell-Mann matrix $\lambda_{a}$ and the gauge coupling $g$. In the NJL sector, $\vec{\tau}$ stands for the isospin matrix, and $G_{\mathrm{S}}$ denotes the coupling constant of the scalar-type fourquark interaction. In $\S 4$, we will add a scalar-type eightquark [25, 29] interaction to the PNJL Lagrangian to discuss the effect on the phase diagram. Effects of eightquark interactions are discussed also in the three flavor NJL model [31]. The Polyakov potential $\mathcal{U}$, defined in (12), is a function of the Polyakov loop $\Phi$ and its complex conjugate $\Phi^{*}$,

$$
\Phi=\frac{1}{N_{\mathrm{c}}} \operatorname{Tr} L, \quad \Phi^{*}=\frac{1}{N_{\mathrm{c}}} \operatorname{Tr} L^{\dagger},
$$

with

$$
L(\mathbf{x})=\mathcal{P} \exp \left[i \int_{0}^{\beta} d \tau A_{4}(\mathbf{x}, \tau)\right]
$$

where $\mathcal{P}$ is the path ordering and $A_{4}=i A_{0}$. In the chiral limit $\left(m_{0}=0\right)$, the Lagrangian density has the exact $S U(2)_{\mathrm{L}} \times S U(2)_{\mathrm{R}} \times U(1)_{\mathrm{v}} \times S U(3)_{\mathrm{c}}$ symmetry. 
The temporal component of the gauge field is diagonal in the flavor space, because the color and the flavor space are completely separated out in the present case. In the Polyakov gauge, $L$ can be written in a diagonal form in the color space [15]:

$$
L=e^{i \beta\left(\phi_{3} \lambda_{3}+\phi_{8} \lambda_{8}\right)}=\operatorname{diag}\left(e^{i \beta \phi_{a}}, e^{i \beta \phi_{b}}, e^{i \beta \phi_{c}}\right),
$$

where $\phi_{a}=\phi_{3}+\phi_{8} / \sqrt{3}, \phi_{b}=-\phi_{3}+\phi_{8} / \sqrt{3}$ and $\phi_{c}=-\left(\phi_{a}+\phi_{b}\right)=-2 \phi_{8} / \sqrt{3}$. The Polyakov loop $\Phi$ is an exact order parameter of the spontaneous $\mathbb{Z}_{3}$ symmetry breaking in the pure gauge theory. Although the $\mathbb{Z}_{3}$ symmetry is not an exact one in the system with dynamical quarks, it still seems to be a good indicator of the deconfinement phase transition. Therefore, we use $\Phi$ to define the deconfinement phase transition.

Under the mean field approximation (MFA), the Lagrangian density becomes

$$
\mathcal{L}_{\mathrm{MFA}}=\bar{q}\left(i \gamma_{\mu} D^{\mu}-\left(m_{0}+\Sigma_{\mathrm{s}}\right)\right) q-U_{\mathrm{M}}(\sigma)-\mathcal{U}\left(\Phi, \Phi^{*}, T\right),
$$

where

$$
\sigma=\langle\bar{q} q\rangle, \quad \Sigma_{\mathrm{s}}=-2 G_{\mathrm{s}} \sigma, \quad U_{\mathrm{M}}=G_{\mathrm{s}} \sigma^{2} .
$$

Using the usual techniques, one can obtain the thermodynamic potential per volume

$$
\begin{aligned}
\Omega= & -\frac{T \ln Z}{V} \\
= & -2 N_{f} \int \frac{d^{3} \mathrm{p}}{(2 \pi)^{3}}\left[3 E(\mathrm{p})+\frac{1}{\beta} \operatorname{Tr}_{\mathrm{c}} \ln \left(1+L e^{-\beta E^{-}(\mathrm{p})}\right)\right. \\
& \left.+\frac{1}{\beta} \operatorname{Tr}_{\mathrm{c}} \ln \left(1+L^{\dagger} e^{-\beta E^{+}(\mathrm{p})}\right)\right]+U_{\mathrm{M}}(\sigma)+\mathcal{U}\left(\Phi, \Phi^{*}, T\right),
\end{aligned}
$$

where $E(\mathrm{p})=\sqrt{\mathrm{p}^{2}+M^{2}}, E^{ \pm}(\mathrm{p})=E(\mathrm{p}) \pm \mu=E(\mathrm{p}) \pm$ $i \theta / \beta$ and $M=m_{0}+\Sigma_{\mathrm{s}}$. In this paper, the thermodynamic potential per volume, $\Omega$, is simply called the thermodynamic potential. After some algebra, the thermodynamic potential $\Omega$ becomes [18]

$$
\begin{aligned}
\Omega= & -2 N_{f} \int \frac{d^{3} \mathrm{p}}{(2 \pi)^{3}}[3 E(\mathrm{p}) \\
& +\frac{1}{\beta} \ln \left[1+3\left(\Phi+\Phi^{*} e^{-\beta E^{-}(\mathbf{p})}\right) e^{-\beta E^{-}(\mathbf{p})}+e^{-3 \beta E^{-}(\mathbf{p})}\right] \\
& \left.+\frac{1}{\beta} \ln \left[1+3\left(\Phi^{*}+\Phi e^{-\beta E^{+}(\mathbf{p})}\right) e^{-\beta E^{+}(\mathbf{p})}+e^{-3 \beta E^{+}(\mathbf{p})}\right]\right] \\
& +U_{\mathrm{M}}+\mathcal{U} .
\end{aligned}
$$

We use $\mathcal{U}$ of Ref. [18] that is fitted to a lattice QCD simulation in the pure gauge theory at finite $T$ [32, 33]:

$$
\begin{aligned}
& \frac{\mathcal{U}}{T^{4}}=-\frac{b_{2}(T)}{2} \Phi^{*} \Phi-\frac{b_{3}}{6}\left(\Phi^{* 3}+\Phi^{3}\right)+\frac{b_{4}}{4}\left(\Phi^{*} \Phi\right)^{2}, \\
& b_{2}(T)=a_{0}+a_{1}\left(\frac{T_{0}}{T}\right)+a_{2}\left(\frac{T_{0}}{T}\right)^{2}+a_{3}\left(\frac{T_{0}}{T}\right)^{3},
\end{aligned}
$$

where parameters are summarized in Table I. The Polyakov potential yields a deconfinement phase transition at $T=T_{0}$ in the pure gauge theory. In the previous paper [30], hence, $T_{0}$ was taken to be $270 \mathrm{MeV}$ predicted by the pure gauge lattice QCD calculation. However, the PNJL model with this value of $T_{0}$ yields somewhat larger value of the transition temperature at zero density than that predicted by the full LQCD simulation [34, 35, 36]. Therefore, we rescale $T_{0}$ in $\S 4$.

\begin{tabular}{cccccc}
\hline$a_{0}$ & $a_{1}$ & $a_{2}$ & $a_{3}$ & $b_{3}$ & $b_{4}$ \\
\hline 6.75 & -1.95 & 2.625 & -7.44 & 0.75 & 7.5 \\
\hline
\end{tabular}

TABLE I: Summary of the parameter set in the Polyakov sector used in Ref. [18]. All parameters are dimensionless.

The variables of $\Phi, \Phi^{*}$ and $\sigma$ satisfy the stationary conditions,

$$
\partial \Omega / \partial \Phi=0, \quad \partial \Omega / \partial \Phi^{*}=0, \quad \partial \Omega / \partial \sigma=0 .
$$

The solutions of the stationary conditions do not give a global minimum $\Omega$ necessarily; there is a possibility that they yield a local minimum or even a maximum. We then search a global minimum directly by varying the variables and check the solutions to satisfy (14). The physical thermodynamic potential $\Omega(\theta)$ at each $\theta$ is obtained by inserting the solutions at each $\theta$ into (11).

\section{EXTENDED $\mathbb{Z}_{3}$ SYMMETRY}

The thermodynamic potential $\Omega$ of Eq. (11) is not invariant under the $\mathbb{Z}_{3}$ transformation,

$$
\Phi(\theta) \rightarrow \Phi(\theta) e^{-i 2 \pi k / 3}, \quad \Phi(\theta)^{*} \rightarrow \Phi(\theta)^{*} e^{i 2 \pi k / 3},
$$

although $\mathcal{U}$ of (12) is invariant. Instead of the $\mathbb{Z}_{3}$ symmetry, however, $\Omega$ is invariant under the extended $\mathbb{Z}_{3}$ transformation,

$$
\begin{aligned}
& e^{ \pm i \theta} \rightarrow e^{ \pm i \theta} e^{ \pm i \frac{2 \pi k}{3}}, \quad \Phi(\theta) \rightarrow \Phi(\theta) e^{-i \frac{2 \pi k}{3}}, \\
& \Phi(\theta)^{*} \rightarrow \Phi(\theta)^{*} e^{i \frac{2 \pi k}{3}} .
\end{aligned}
$$

It is convenient to introduce the modified Polyakov loop $\Psi \equiv e^{i \theta} \Phi$ and $\Psi^{*} \equiv e^{-i \theta} \Phi^{*}$ invariant under the transformation (16). The extended $\mathbb{Z}_{3}$ transformation is then rewritten into

$$
\begin{aligned}
& e^{ \pm i \theta} \rightarrow e^{ \pm i \theta} e^{ \pm i \frac{2 \pi k}{3}}, \quad \Psi(\theta) \rightarrow \Psi(\theta), \\
& \Psi(\theta)^{*} \rightarrow \Psi(\theta)^{*},
\end{aligned}
$$


and $\Omega$ is also into

$$
\begin{aligned}
\Omega= & -2 N_{f} \int \frac{d^{3} \mathrm{p}}{(2 \pi)^{3}}\left[3 E(\mathrm{p})+\frac{1}{\beta} \ln \left[1+3 \Psi e^{-\beta E(\mathbf{p})}\right.\right. \\
& \left.+3 \Psi^{*} e^{-2 \beta E(\mathbf{p})} e^{\beta \mu_{\mathrm{B}}}+e^{-3 \beta E(\mathbf{p})} e^{\beta \mu_{\mathrm{B}}}\right] \\
& +\frac{1}{\beta} \ln \left[1+3 \Psi^{*} e^{-\beta E(\mathbf{p})}+3 \Psi e^{-2 \beta E(\mathbf{p})} e^{-\beta \mu_{\mathrm{B}}}\right. \\
& \left.\left.+e^{-3 \beta E(\mathbf{p})} e^{-\beta \mu_{\mathrm{B}}}\right]\right]+U_{\mathrm{M}}+\left[-\frac{b_{2}(T) T^{4}}{2} \Psi^{*} \Psi\right. \\
& \left.-\frac{b_{3} T^{4}}{6}\left(\Psi^{* 3} e^{\beta \mu_{\mathrm{B}}}+\Psi^{3} e^{-\beta \mu_{\mathrm{B}}}\right)+\frac{b_{4} T^{4}}{4}\left(\Psi^{*} \Psi\right)^{2}\right],
\end{aligned}
$$

where $\mu_{\mathrm{B}}=3 \mu=i 3 \theta / \beta$ is the baryonic chemical potential and the factor $e^{ \pm \beta \mu_{\mathrm{B}}}$ is invariant under the transformation (17). Obviously, $\Omega$ is invariant under the transformation (17).

As a feature of the extended $\mathbb{Z}_{3}$ transformation (17), the external parameter $\mu=i \theta / \beta$ varies with (17), and the modified Polyakov-loop is invariant under (17) and then not an order parameter of the extended $\mathbb{Z}_{3}$ symmetry. The same is seen for the chiral transformation in the chiral perturbation theory [37, 38]. The Lagrangian of the theory is

$$
\begin{aligned}
L_{\mathrm{chPT}}= & \frac{1}{16} F^{2} \operatorname{Tr}\left\{\partial_{\mu} U_{\mathrm{NG}} \partial^{\mu} U_{\mathrm{NG}}^{\dagger}\right\} \\
& +\frac{1}{2} v \operatorname{Tr}\left\{M_{\mathrm{q}}\left(U_{\mathrm{NG}}^{\dagger}+U_{\mathrm{NG}}\right)\right\}+\cdots
\end{aligned}
$$

with

$$
U_{\mathrm{NG}}=\exp \left(2 i \sum_{a=1}^{8} \xi_{a} \lambda^{a}\right)
$$

where $F^{2}$ is a positive parameter, and $-v, \xi_{a}$ and $M_{\mathrm{q}}$ represent, the expectation value of the quark bilinear, the Nambu-Goldstone boson fields and the real matrix for quark masses in three flavor space, respectively. The Lagrangian (19) is not invariant under the chiral transformation

$$
U_{\mathrm{NG}} \rightarrow \exp \left(i \sum_{a=1}^{8} \theta_{a}^{\mathrm{R}} \lambda_{a}\right) U_{\mathrm{NG}} \exp \left(-i \sum_{a=1}^{8} \theta_{a}^{\mathrm{L}} \lambda_{a}\right),
$$

since the second term including $M_{\mathrm{q}}$ in the Lagrangian breaks the symmetry explicitly, where $\theta_{a}^{\mathrm{L}}$ and $\theta_{a}^{\mathrm{R}}$ are arbitrary real parameters of the transformation for the leftand right-handed quark fields. However, once the quark mass matrix $M_{\mathrm{q}}$ is assumed to be transformed as

$$
M_{\mathrm{q}} \rightarrow \exp \left(i \sum_{a=1}^{8} \theta_{a}^{\mathrm{R}} \lambda_{a}\right) M_{\mathrm{q}} \exp \left(-i \sum_{a=1}^{8} \theta_{a}^{\mathrm{L}} \lambda_{a}\right)
$$

the Lagrangian (19) becomes invariant under the chiral transformation, and $v$ is not an order parameter of the extended chiral symmetry anymore.
Although we have no order parameter for the extended $\mathbb{Z}_{3}$ symmetry, this symmetry leads many useful conclusions [30] as shown below. Under the transformation $\theta \rightarrow \theta+2 \pi k / 3$, (18) keeps the same form, if $\Psi(\theta)$ and $\Psi(\theta)^{*}$ are replaced by $\Psi(\theta+2 \pi k / 3)$ and $\Psi(\theta+2 \pi k / 3)^{*}$, respectively. This means that the stationary conditions for $\Psi(\theta)$ and $\Psi(\theta)^{*}$ agree with those for $\Psi(\theta+2 \pi k / 3)$ and $\Psi(\theta+2 \pi k / 3)^{*}$, respectively, and then that

$$
\Psi\left(\theta+\frac{2 \pi k}{3}\right)=\Psi(\theta) \text { and } \Psi\left(\theta+\frac{2 \pi k}{3}\right)^{*}=\Psi(\theta)^{*}
$$

The potential $\Omega$ depends on $\theta$ through $\Psi(\theta), \Psi(\theta)^{*}$, $\sigma(\theta)$ and $e^{3 i \theta}$. We then denote $\Omega(\theta)$ by $\Omega(\theta)=$ $\Omega\left(\Psi(\theta), \Psi(\theta)^{*}, e^{3 i \theta}\right)$, where $\sigma(\theta)$ is suppressed since it is irrelevant to discussion shown below. The RW periodicity of $\Omega$ is then shown as

$$
\begin{aligned}
\Omega\left(\theta+\frac{2 \pi k}{3}\right) & =\Omega\left(\Psi\left(\theta+\frac{2 \pi k}{3}\right), \Psi\left(\theta+\frac{2 \pi k}{3}\right)^{*}, e^{3 i\left(\theta+\frac{2 \pi k}{3}\right)}\right) \\
& =\Omega(\theta),
\end{aligned}
$$

by using (23) in the second equality.

Equation (18) keeps the same form under the transformation $\theta \rightarrow-\theta$, if $\Psi(\theta)$ and $\Psi(\theta)^{*}$ are replaced by $\Psi(-\theta)^{*}$ and $\Psi(-\theta)$, respectively. This indicates that

$$
\Psi(-\theta)=\Psi(\theta)^{*} \quad \text { and } \quad \Psi(-\theta)^{*}=\Psi(\theta) .
$$

Furthermore, $\Omega$ is a real function, as shown in (18). Using these properties, one can show that

$$
\begin{aligned}
\Omega(\theta) & =(\Omega(\theta))^{*}=\Omega\left(\Psi(\theta)^{*}, \Psi(\theta), e^{-3 i \theta}\right) \\
& =\Omega\left(\Psi(-\theta), \Psi(-\theta)^{*}, e^{-3 i \theta}\right)=\Omega(-\theta) .
\end{aligned}
$$

Thus, $\Omega$ is a periodic even function of $\theta$ with a period $2 \pi / 3$. The chiral condensate $\sigma(\theta)$ is also a periodic even function of $\theta, \sigma(\theta)=\sigma(\theta+2 \pi k / 3)=\sigma(-\theta)$, because it is given by $\sigma(\theta)=d \Omega(\theta) / d m_{0}$. Furthermore, the quark number density $\rho_{\mathrm{v}}=-d \Omega / d(i T \theta)$ is pure imaginary and a periodic odd function of $\theta$.

The modified Polyakov loop $\Psi$ has a periodicity of (23). The real (imaginary) part of $\Psi$ is even (odd) under the interchange $\theta \leftrightarrow-\theta$, because

$$
\begin{aligned}
\operatorname{Re}[\Psi(\theta)] & =\left(\Psi(\theta)+\Psi(\theta)^{*}\right) / 2=\operatorname{Re}[\Psi(-\theta)], \\
\operatorname{Im}[\Psi(\theta)] & =\left(\Psi(\theta)-\Psi(\theta)^{*}\right) /(2 i)=-\operatorname{Im}[\Psi(-\theta)],
\end{aligned}
$$

where use has been made of (25). Thus, the real (imaginary) part of $\Psi$ is a periodic even (odd) function of $\theta$. Similarly, the absolute value $|\Psi|$ (phase $\phi$ ) of the Polyakov loop is a periodic even (odd) function of $\theta$, because $|\Psi|=\sqrt{(\operatorname{Re}[\Psi])^{2}+(\operatorname{Im}[\Psi])^{2}}(\phi=$ $\arctan (\operatorname{Im}[\Psi] / \operatorname{Re}[\Psi]))$.

Since $\Omega(\theta), \Psi(\theta)$ and $\sigma(\theta)$ are periodic functions of $\theta$ with a period $2 \pi / 3$, here we think a period $0 \leq \theta \leq 2 \pi / 3$. In the region, periodic even functions such as $\Omega(\theta), \sigma(\theta)$, $\operatorname{Re}[\Psi(\theta)]$ and $|\Psi|$ are symmetric with respect to a line $\theta=\pi / 3$. This indicates that such an even function has a 
cusp at $\theta=\pi / 3$, if the gradient $d \Omega / d \theta$ is not zero. Such a cusp comes out in the high $T$ region, as shown in $\S 4$ with numerical calculations. This means that the chiral RW phase transition at $\theta=\pi / 3$ is second order.

Meanwhile, $\operatorname{Im}[\Psi(\theta)], \phi$ and $\rho_{\mathrm{v}}$ are periodic odd functions of $\theta$. This leads to the fact that these are discontinuous at $\theta=\pi / 3$, if the odd functions are not zero there. Thus, the Polyakov-loop RW phase transition appears as first order in $\operatorname{Im}[\Psi(\theta)]$ and $\phi$ and as second order in $\operatorname{Re}[\Psi(\theta)]$ and $|\Psi|$. The RW transition appearing in $\rho_{\mathrm{v}}$ at $\theta=\pi / 3$ is also first order. These are seen in the high $T$ region, as shown in $\S 4$. The orders of the Polyakovloop and chiral RW phase transitions and the RW transition appearing in $\rho_{\mathrm{v}}$ are not affected by the existence of the current quark mass and multi-quark interactions introduced later, since the periodicity and the odd/even property of the physical quantities are not changed.

The dynamical variables $\Psi$ and $\Psi^{*}$ are also invariant under the continuous phase transformation,

$$
e^{ \pm i \theta} \rightarrow e^{ \pm i \theta} e^{ \pm i \alpha}, \quad \Phi \rightarrow \Phi e^{-i \alpha}, \quad \Phi^{*} \rightarrow \Phi^{*} e^{i \alpha}
$$

for an arbitrary real parameter $\alpha$. However, the factor $e^{ \pm \beta \mu_{\mathrm{B}}}\left(=e^{ \pm 3 i \theta}\right)$ and the potential $\Omega$ including the factor are not invariant. If $\Omega$ were invariant under (27), the continuous symmetry would lead to a simple relation $\Psi(\theta+\alpha)=\Psi(\theta)$, that is $\Phi(\theta+\alpha)=e^{-i \alpha} \Phi(\theta)$, that guarantees that $\Phi$ is a smooth function of $\theta$. When $T$ is small under the condition that $\mu$ is imaginary and $\Psi$ and $\Psi^{*}$ are not zero, the thermodynamic potential (18) is reduced to

$$
\begin{aligned}
\Omega & \sim-2 N_{f} \int \frac{d^{3} \mathrm{p}}{(2 \pi)^{3}}\left[3 E(\mathrm{p})+\frac{1}{\beta} \ln \left[1+3 \Psi e^{-\beta E(\mathbf{p})}\right]\right. \\
& \left.+\frac{1}{\beta} \ln \left[1+3 \Psi^{*} e^{-\beta E(\mathbf{p})}\right]\right]+U_{\mathrm{M}}-\frac{a_{3} T_{0}^{3} T}{2} \Psi^{*} \Psi
\end{aligned}
$$

This has no explicit $\mu_{\mathrm{B}}(=3 i \theta T)$ dependence. Therefore, at low temperature, $\Omega$ is approximately invariant under (27) and $\Phi$ can rotate smoothly as $\theta$ varies. At high temperature, however, effects of the explicit $\mu_{\mathrm{B}}$ dependence are not negligible and $\Phi$ can not rotate smoothly. Thus, it is obvious that the Polyakov-loop RW phase transition at high $T$ is originated in the factor $e^{ \pm \beta \mu_{\mathrm{B}}}$ in (18). At high temperature, the continuous symmetry under the transformation (27) is broken into a discrete symmetry, i.e., the extended $\mathbb{Z}_{3}$ symmetry, through the factor $e^{ \pm \beta \mu_{\mathrm{B}}}$.

It is easily seen that the ordinary NJL model respects the chiral symmetry but it does not preserve the extended $\mathbb{Z}_{3}$ symmetry. On the contrary, the 3-dimensional 3-state Potts model [39, 40, 41, 42] respects the extended $\mathbb{Z}_{3}$ symmetry and then has the RW periodicity, but it does not possess the chiral symmetry, since the model is a paradigm of QCD in the large quark mass limit. In lattice QCD (LQCD), to avoid the quadratic divergence, the chemical potential should be introduced just like the fourth component of an imaginary constant vector potential [43], i.e., $e^{\mu a} U_{4}$ or $e^{-\mu a} U_{4}^{\dagger}$, where $a$ and $U_{4}\left(=e^{i a A_{4}}\right)$ are the lattice spacing and the fourth component of gauge field on lattice, respectively. In this case, the RW periodicity is expected to be naturally satisfied. In Table II, we summarize these symmetry properties in the three effective models together with QCD and LQCD. Among the effective models, only the PNJL model has the same properties as QCD does.

\begin{tabular}{ccccc}
\hline Theory & Chiral & $\mathbb{Z}_{3}$ & extended $\mathbb{Z}_{3}$ & RW periodicity \\
\hline QCD & $\mathrm{P}$ & $\mathrm{PP}$ & $\mathrm{P}$ & $\mathrm{P}$ \\
\hline LQCD & $\mathrm{P}$ & $\mathrm{PP}$ & $\mathrm{P}$ & $\mathrm{P}$ \\
\hline NJL & $\mathrm{P}$ & $\mathrm{ND}$ & $\mathrm{B}$ & $\mathrm{B}$ \\
\hline PNJL & $\mathrm{P}$ & $\mathrm{PP}$ & $\mathrm{P}$ & $\mathrm{P}$ \\
\hline 3-d 3-state Potts & $\mathrm{B}$ & $\mathrm{PP}$ & $\mathrm{P}$ & $\mathrm{P}$ \\
\hline
\end{tabular}

TABLE II: Summary of symmetry properties in several theories. In QCD, LQCD, NJL and PNJL, we consider the chiral limit, $m_{0}=0 ; \mathrm{P}$ means "preserved", $\mathrm{PP}$ does "preserved in the pure gauge sector", B does "broken explicitly" and ND does "not defined".

Although the original NJL model does not has the extended $\mathbb{Z}_{3}$ symmetry, it may be a good approximation to the PNJL model. In the mean field approximation, the fermionic part $\Omega_{\mathrm{NJL}}^{\mathrm{f}}$ of the NJL thermodynamic potential is given by

$$
\begin{aligned}
\Omega_{\mathrm{NJL}}^{\mathrm{f}}= & -2 N_{f} N_{c} \int \frac{d^{3} \mathrm{p}}{(2 \pi)^{3}}[E(\mathrm{p}) \\
& \left.+\frac{1}{\beta} \ln \left[1+e^{-\beta E^{-}(\mathbf{p})}\right]+\frac{1}{\beta} \ln \left[1+e^{-\beta E^{+}(\mathbf{p})}\right]\right] .
\end{aligned}
$$

It is easily seen that $\Omega_{\mathrm{PNJL}}^{\mathrm{f}}\left(\Phi=\Phi^{*}=1\right)=\Omega_{\mathrm{NJL}}^{\mathrm{f}}$. Therefore, the fermionic part of the NJL thermodynamic potential coincides with that of the PNJL model in the perfectly deconfinement phase. In the confinement phase, meanwhile, $\Omega_{\mathrm{PNJL}}^{\mathrm{f}}\left(\Phi=\Phi^{*}=0\right)=\Omega_{\mathrm{NJL}}^{\mathrm{f}}$ is not held in general. Actually, it is also easily shown that

$$
\begin{aligned}
& \Omega_{\mathrm{PNJL}}^{\mathrm{f}}\left(\Phi=\Phi^{*}=0\right)= \\
& \frac{\Omega_{\mathrm{NJL}}^{\mathrm{f}}(T, \mu)+\Omega_{\mathrm{NJL}}^{\mathrm{f}}\left(T, \mu+\frac{2 \pi i}{3} T\right)+\Omega_{\mathrm{NJL}}^{\mathrm{f}}\left(T, \mu-\frac{2 \pi i}{3} T\right)}{N} .
\end{aligned}
$$

Therefore, in the confined phase, the fermionic part of the PNJL thermodynamic potential does not coincide with $\Omega_{\mathrm{NJL}}^{\mathrm{f}}$ but with the $\mathbb{Z}_{3}$ symmetrized average of $\Omega_{\mathrm{NJL}}^{\mathrm{f}}$. However, in the zero temperature limit, the thermodynamic potential of the PNJL model is reduced to that of the NJL model as

$$
\begin{aligned}
& \Omega_{\mathrm{PNJL}}(T=0) \\
& =-6 N_{f} \int \frac{d^{3} \mathrm{p}}{(2 \pi)^{3}}\left[E(\mathbf{p})-\theta\left(-E^{-}(\mathbf{p})\right) E^{-}(\mathbf{p})\right] \\
& \quad+U_{\mathrm{M}}\left(\sigma, \rho_{\mathrm{v}}(T \rightarrow 0, \mu, \sigma)\right)+\mathcal{U}\left(T \rightarrow 0, \Phi, \Phi^{*}\right) \\
& =\Omega_{\mathrm{NJL}}(T=0) .
\end{aligned}
$$


Therefore, the difference between the NJL model and the PNJL model is significant only in the intermediate temperature region. In both the high and the low temperature region, the two model give similar results for the physics concerning the chiral symmetry. It should be also remarked that the vacuum term in the PNJL thermodynamic potential (11) is the same as that of the NJL model. Therefore, the parameters included in the NJL sector of the PNJL model are the same as the parameters in the original NJL model, if they are determined phenomenologically at $T=\mu=0$. This also ensures that the original NJL model is a good approximation to the PNJL model. Thus, the difference between the two models is significant in the intermediate temperature region. Actually, the PNJL model makes the critical endpoint [27, 28, 29] shift to higher $T$ and lower $\mu$ than the NJL model does [21, 25].

\section{NUMERICAL RESULTS}

Since the NJL model is nonrenormalizable, it is then needed to introduce a cutoff in the momentum integration. Here we take the three-dimensional momentum cutoff

$$
\int \frac{d^{3} \mathbf{p}}{(2 \pi)^{3}} \rightarrow \frac{1}{2 \pi^{2}} \int_{0}^{\Lambda} d p p^{2}
$$

Hence, the present model has three parameters $m_{0}, \Lambda$, $G_{\mathrm{s}}$ in the NJL sector. Following Ref. [29], we use $\Lambda=$ $0.6315 \mathrm{GeV}$ and $G_{\mathrm{s}}=5.498 \mathrm{GeV}^{-2}$, but vary $m_{0}$ as a free parameter. If we put $m_{0}=5.5 \mathrm{MeV}$, these parameters reproduce the pion decay constant $f_{\pi}=93.3 \mathrm{MeV}$ and the pion mass $M_{\pi}=138 \mathrm{MeV}$.

Figure 2 shows the $T$ dependence of the chiral condensate $\sigma$ and the absolute value of the modified Polyakov loop, $|\Psi|$, at $\theta=0$ for three cases of $T_{0}$. The quantity $|\Psi|(\sigma)$ indicates that a crossover transition takes place at $T_{\mathrm{D}}=240 \mathrm{MeV}\left(T_{\mathrm{C}}=261 \mathrm{MeV}\right)$ for $T_{0}=270 \mathrm{MeV}$ and at $T_{\mathrm{D}}=176 \mathrm{MeV}\left(T_{\mathrm{C}}=221 \mathrm{MeV}\right)$ for $T_{0}=190 \mathrm{MeV}$. We took the original value $T_{0}=270 \mathrm{MeV}$ in the previous work [30], but in this paper we take the rescaled one $T_{0}=190 \mathrm{MeV}$. In this case the values of $T_{\mathrm{C}}$ and $T_{\mathrm{D}}$ are closer to $170 \sim 180 \mathrm{MeV}$, the results of the two-flavor full LQCD simulation [34, 35, 36], than those in the case of $T_{0}=270 \mathrm{MeV}$, although the difference $T_{\mathrm{C}}-T_{\mathrm{D}}$ becomes larger as $T_{0}$ decreases, as shown in Fig. 2.

Figure 3 shows the $T$ dependence of the chiral condensate and the modified Polyakov loop for three cases of $m_{0}$. In the chiral limit $m_{0}=0$, the chiral condensate shows that a second-order chiral phase transition takes place around $230 \mathrm{MeV}$ not only for $\theta=0$ but also for $\theta \neq 0$. The transition becomes crossover when $m_{0}$ is finite. Meanwhile, it is found from the modified Polyakov loop that the crossover deconfinement transition that appears around $180 \mathrm{MeV}$ becomes sharper as $m_{0}$ increases. This behavior is consistent with the result of the 3-dimensional 3-state Potts model [39, 40, 41, 42] in

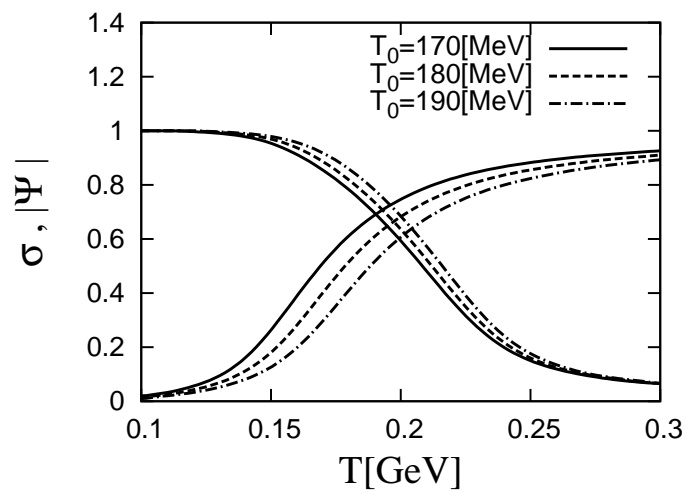

Fig. 2: $T$ dependence of the chiral condensate normalized by $\sigma(T=0, \mu=0)$ and the absolute value of the modified Polyakov loop $\Psi(\theta)$ at $\theta=0$ for three cases of $T_{0}$. Increasing (decreasing) functions of $\theta$ denote $|\Psi|(\sigma)$ for all the cases.

which a first-order deconfinement transition is observed for large quark mass.
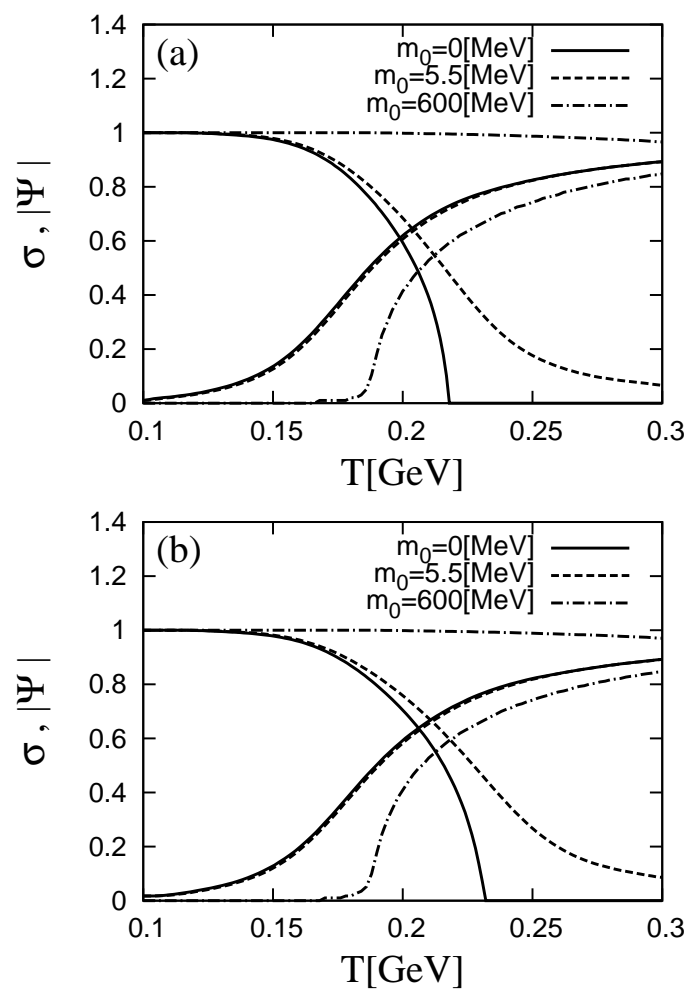

Fig. 3: The chiral condensate $\sigma$ normalized by $\sigma(T=0, \mu=$ 0 ) and the absolute value of $\Psi(\theta)$ as a function of $T$ for three cases of $m_{0}$; (a) corresponds to $\theta=0$ and (b) to $\theta=\frac{\pi}{6}$. Increasing (decreasing) functions of $T$ denote $|\Psi|(\sigma)$ for all the cases.

Figure 4 shows the thermodynamic potential $\Omega$ as a function of $\theta$ in two cases of $T=170$ and $200 \mathrm{MeV}$. The potential $\Omega$ is smooth everywhere in the low $T$ case, 
but not at $\theta=(2 k+1) \pi / 3$ in the high $T$ case. This result is consistent with the RW prediction [11] and lattice simulation [7] on the $\theta$ and the $T$ dependence of the QCD thermodynamic potential. Qualitative features of $\Omega$ at $\theta=(2 k+1) \pi / 3$ are the same as in the chiral limit case [30].

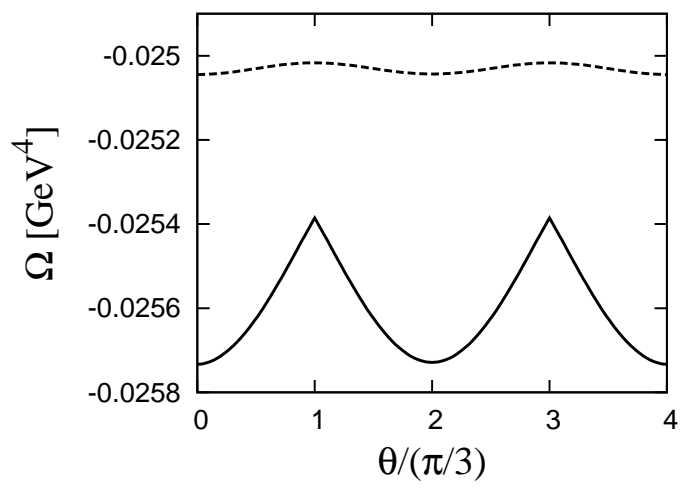

Fig. 4: Thermodynamic potential $\Omega\left(\right.$ in $\mathrm{GeV}^{4}$ ) as a function of $\theta$. The dashed line corresponds to the case of $T=170 \mathrm{MeV}$ and the solid one to that of $T=200 \mathrm{MeV}$.

Figure 5 shows the real and imaginary parts of $\Psi(\theta)$ as a function of $\theta$. In the case of $T=200 \mathrm{MeV}$, the imaginary part of $\Psi(\theta)$ is discontinuous at $\theta=(2 k+$ 1) $\pi / 3$, while the real part of $\Psi(\theta)$ is continuous but not smooth there. The transition appearing at $\theta=(2 k+$ 1) $\pi / 3$ is the Polyakov loop RW phase transition. In the case of $T=170 \mathrm{MeV}$, meanwhile, both the real and the imaginary part are smooth everywhere.

Figure 6 shows the absolute value $|\Psi|$ and the phase $\phi$ of the modified Polyakov loop as a function of $\theta$. In the case of $T=200 \mathrm{MeV}$, the phase $\phi(\theta)$ is discontinuous at $\theta=(2 k+1) \pi / 3$, while the absolute value $|\Psi(\theta)|$ is continuous but not smooth there. In the case of $T=$ $170 \mathrm{MeV}$, meanwhile, both the absolute value and the phase are smooth everywhere. All the results on the $\theta$ and the $T$ dependence of $\Psi$ are consistent with the results of lattice simulations [3, 4, 5, 6, 7]. As an interesting feature, the transition appears as first order in the phase $\phi$ and the imaginary part of $\Psi(\theta)$ and as second order in the absolute value and the real part of $\Psi(\theta)$. Qualitative features of the Polyakov-loop RW transition are the same as in the chiral limit [30].

Figure 7 shows the chiral condensate $\sigma$ as a function of $\theta$. In the case of $T=200 \mathrm{MeV}, \sigma$ has a cusp at each of lines $\theta=(2 k+1) \pi / 3$. Thus, the chiral phase transition of second order comes out at $\theta=(2 k+1) \pi / 3$. Meanwhile, in the case of $T=170 \mathrm{MeV}$, there is no cusp at $\theta=(2 k+1) \pi / 3$, indicating no chiral phase transition there. As a feature not seen in the chiral limit, $\sigma$ and the cusp do not vanish even in much higher temperature than $T=200 \mathrm{MeV}$.

Figure 8 shows the imaginary part of quark number density $\rho_{\mathrm{v}}$ as a function $\theta$; note that the real part is
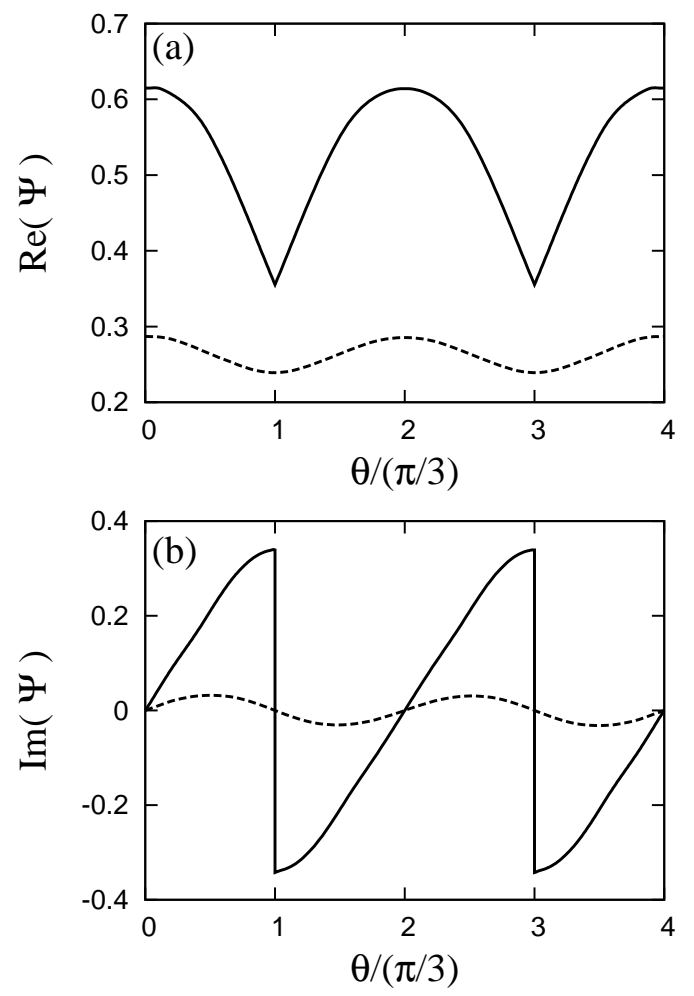

Fig. 5: The modified Polyakov loop $\Psi(\theta)$ as a function of $\theta$; (a) for the real part and (b) for the imaginary part. Definitions of lines are the same as in Fig. 4.

always zero. In the case of $T=200 \mathrm{MeV}$, it is discontinuous at $\theta=(2 k+1) \pi / 3$, indicating that the phase transition is first order. In the case of $T=200 \mathrm{MeV}, \rho_{\mathrm{v}}$ is smooth everywhere. The $\theta$ and the $T$ dependence of $\rho_{\mathrm{v}}$ are consistent with the results of lattice simulations [4]. Comparing Fig. 8 with Fig. 5 (b), we see that the imaginary part of the quark number density has $\theta$ dependence similar to the imaginary part of the modified Polyakov loop. This is natural, because the former is related to the fourth component of the vector current, while the latter to the fourth component of the vector field.

Thus, the PNJL results are consistent with the lattice ones [4, [, , 6], except that the temperature difference $T_{\mathrm{C}}-T_{\mathrm{D}}$ is considerably large in the former but small in the latter. Since the lattice simulations made in the imaginary $\mu$ region have small lattice sizes, the results are not conclusive necessarily. However, it is important to check whether the PNJL model can reproduce the present lattice data in the finite imaginary $\mu$ region. This is discussed below from a qualitative point of view.

Our calculations have no free parameter, since the parameters of the Polyakov-potential sector have been fixed to reproduce the results of the lattice QCD simulations and the other parameters in the NJL sector have been adjusted to reproduce the empirical values of $f_{\pi}$ and $M_{\pi}$, i.e., $f_{\pi}=93.3 \mathrm{MeV}$ and $M_{\pi}=138 \mathrm{MeV}$. Therefore, we need a new parameter to improve this situation. It is 

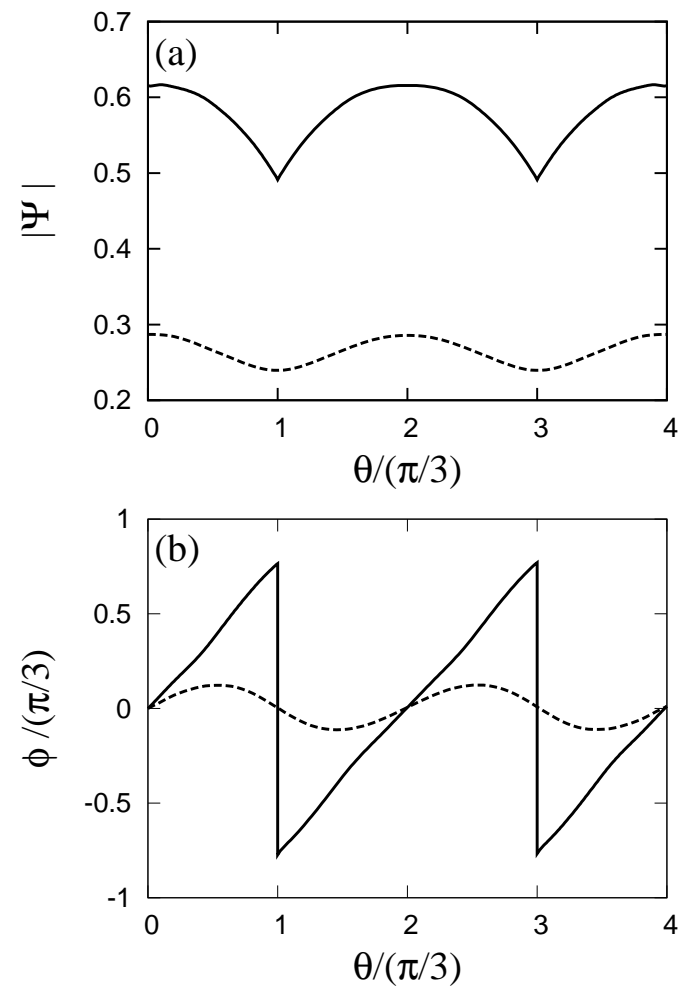

Fig. 6: The modified Polyakov loop $\Psi(\theta)$ as a function of $\theta$; (a) for the absolute value and (b) for the phase. Definitions of lines are the same as in Fig. 4.

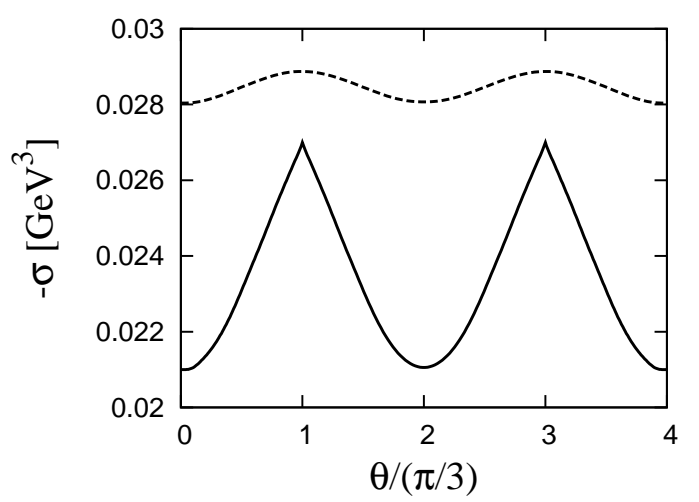

Fig. 7: Chiral condensate $\sigma$ (in $\mathrm{GeV}^{3}$ ) as a function of $\theta$. Definitions of lines are the same as in Fig. 4.

reported that the value of the critical temperature is sensitive to the strength of scalar-type eight-quark interaction in both the NJL model [29] and the PNJL one 25]. Furthermore, the NJL and PNJL models with the eightquark interaction can reproduce lighter $\sigma$-meson mass that may be phenomenologically more favorable [25, 29]. Therefore, we can consider the scalar-type eight-quark interaction as a reasonable extension of the ordinary PNJL model.

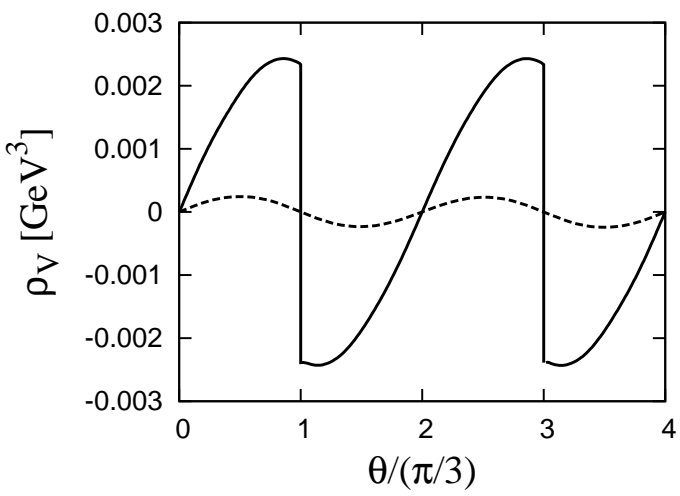

Fig. 8: Imaginary part of the quark number density $\rho_{\mathrm{v}}$ (in $\mathrm{GeV}^{3}$ ) as a function of $\theta$. Definitions of lines are the same as in Fig. 4.

We add the eight-quark interaction [25, 29]

$$
G_{\mathrm{s} 8}\left[(\bar{q} q)^{2}+\left(\bar{q} i \gamma_{5} \vec{\tau} q\right)^{2}\right]^{2}
$$

to Lagrangian (4). The scalar part $\Sigma_{\mathrm{s}}$ of the quark selfenergy and the mesonic potential $U_{\mathrm{M}}$ are modified into

$$
\Sigma_{\mathrm{s}}=-2 G_{\mathrm{s}} \sigma-4 G_{\mathrm{s} 8} \sigma^{3}, \quad U_{\mathrm{M}}=G_{\mathrm{s}} \sigma^{2}+3 G_{\mathrm{s} 8} \sigma^{4} .
$$

Figure 9 shows the $T$ dependence of the chiral condensate and the modified Polyakov loop with and without the eight-quark interaction. We set the parameter $G_{\mathrm{s} 8}$ to $270.9 \mathrm{GeV}^{-8}$ to reproduce $m_{\sigma}=600 \mathrm{MeV}$ at $T=\theta=0$ [29], where $m_{\sigma}$ is the $\sigma$-meson mass. We see for $\theta=0$ that the eight-quark interaction makes the chiral transition temperature $T_{\mathrm{C}}$ shift down to the deconfinement transition temperature $T_{\mathrm{D}} \sim 180 \mathrm{MeV}$. Similar effect is seen also for $\theta=\frac{\pi}{6}$.

Figure 10 represents the phase diagram in the $\theta-T$ plane in the case of $m_{0}=0$. The phase diagram is symmetric with respect to each of lines $\theta=k \pi / 3$ for any integer $k$. The dashed curve between $\mathrm{D}$ and $\mathrm{E}$ represents the deconfinement transition of crossover, and the dotdashed curve between $\mathrm{C}$ and $\mathrm{F}$ does the second-order chiral phase transition. For $\theta \neq k \pi / 3$, thus, the chiral phase transition occurs at higher $T$ than the deconfinement transition does. The solid vertical line starting from point E represents the Polyakov-loop RW phase transition. The chiral RW transition of second order occurs on the line between E and F. Above F, the chiral condensate is zero. Point $\mathrm{F}$ turns out to be a bifurcation of the chiral phase transition line, and point $\mathrm{E}$ is the endpoint of both the Polyakov-loop RW and chiral RW transitions. The chiral phase transition line between $\mathrm{C}$ and $\mathrm{F}$ is shifted down to the vicinity of the deconfinement transition line between $\mathrm{D}$ and $\mathrm{E}$ by adding the eight-quark interaction. In the case without (with) the eight-quark interaction, the temperatures at the point $\mathrm{C}$, $\mathrm{D}, \mathrm{E}, \mathrm{F}$ are $T_{\mathrm{C}}=221(188) \mathrm{MeV}, T_{\mathrm{D}}=172(172) \mathrm{MeV}$, $T_{\mathrm{E}}=190(190) \mathrm{MeV}$ and $T_{\mathrm{F}}=280(236) \mathrm{MeV}$, respectively. 

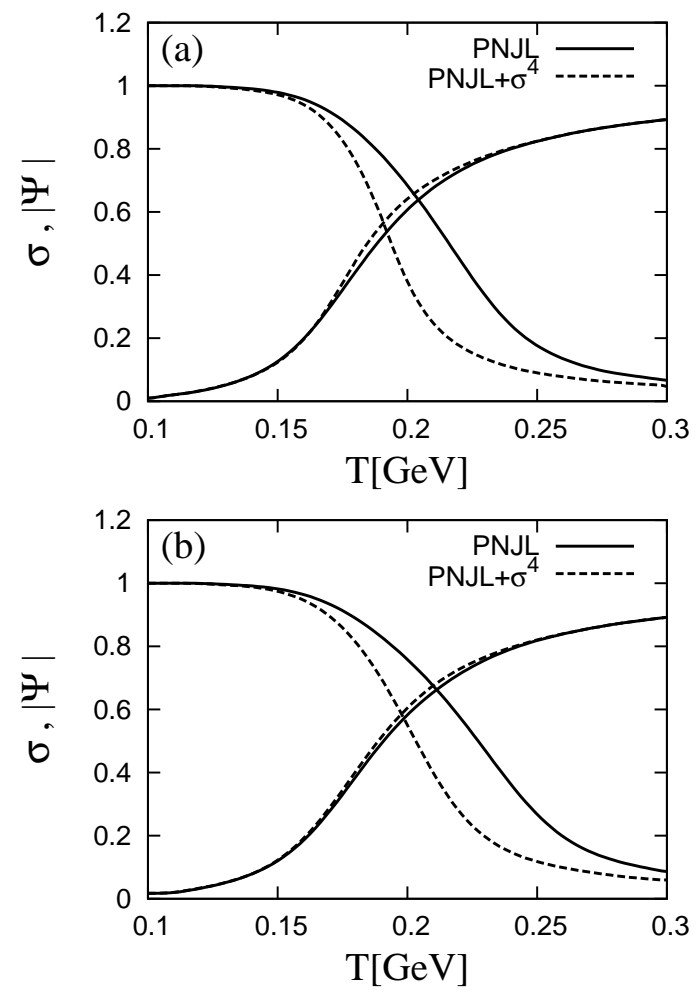

Fig. 9: The chiral condensate $\sigma$ normalized by $\sigma(T=0, \mu=$ 0 ) and the absolute value of $\Psi(\theta)$ as a function of $T$ with and without the eight-quark interaction; (a) for $\theta=0$ and (b) for $\theta=\frac{\pi}{6}$. Increasing (decreasing) functions of $\theta$ denote $|\Psi|(\sigma)$ for all the cases.

Figure 11 represents the phase diagram in the $\theta-T$ plane in the case of $m_{0}=5.5 \mathrm{MeV}$. Again, the phase diagram is symmetric with respect to each of lines $\theta=k \pi / 3$. The dashed curve between $\mathrm{D}$ and $\mathrm{E}$ represents the deconfinement transition of crossover, and the dot-dashed curve between $\mathrm{C}$ and $\mathrm{F}$ does the chiral transition of crossover. Also for this finite $m_{0}$ case, the chiral transition occurs at higher $T$ than the deconfinement transition. The solid vertical line starting from point E represents both the Polyakov-loop RW phase transition and the chiral RW phase transition of second order. Point E is the endpoint of both the RW transitions. As a viewpoint different from the $m_{0}=0$ case [30], point $\mathrm{F}$ is neither a bifurcation of the chiral phase transition line nor an endpoint of the chiral RW phase transition, since the chiral condensate $\sigma$ is always finite on the vertical line $\theta=\pi / 3$, that is, also on the line above point F. Just as in the case of $m_{0}=0$, the chiral transition line between $\mathrm{C}$ and $\mathrm{F}$ is shifted down to the vicinity of the deconfinement transition line between $\mathrm{D}$ and $\mathrm{E}$ by adding the eight-quark interaction. In the case without (with) the eight-quark interaction, the temperatures at the point $\mathrm{C}$, $\mathrm{D}, \mathrm{E}, \mathrm{F}$ are $T_{\mathrm{C}}=218(194) \mathrm{MeV}, T_{\mathrm{D}}=176(176) \mathrm{MeV}$, $T_{\mathrm{E}}=190(190) \mathrm{MeV}$ and $T_{\mathrm{F}}=282(244) \mathrm{MeV}$, respectively.
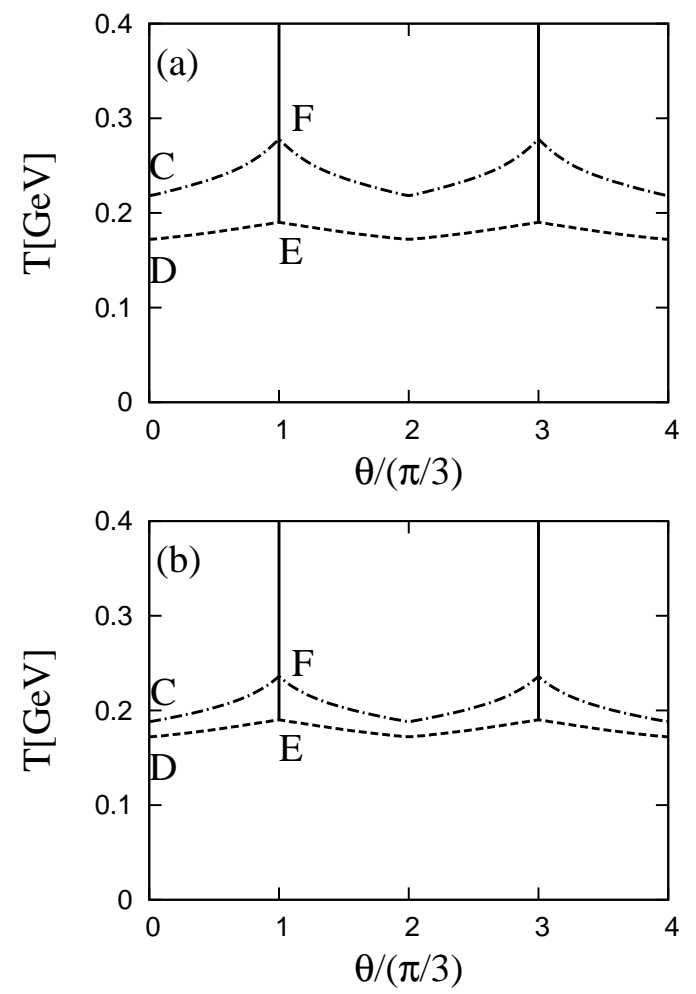

Fig. 10: The phase diagram in the $\theta-T$ plane in the case of $m_{0}=0$; the eight-quark interaction is switched off in (a) and on in (b). The solid vertical line starting from point E represents the Polyakov-loop RW phase transition. The dashed curve between D and E represents the deconfinement transition of crossover, and the dot-dashed curve between $\mathrm{C}$ and $\mathrm{F}$ does the second-order chiral phase transition. The second-order chiral RW transition occurs on the line between $\mathrm{E}$ and $\mathrm{F}$.

Finally, we make a brief trial of the PNJL extrapolation, that is, the lattice data obtained in the imaginary $\mu$ region is extrapolated with PNJL to the real $\mu$ region. The absolute value of the Polaykov loop, $|\Phi|$, is measured for the two-flavor case in Ref. [6], although the lattice simulation of Wilson dynamical quarks has a small size of $8^{3} \times 4$. As the first step, we try to reproduce the lattice data [6] with PNJL. The screening mass of quark is estimated to be a few hundreds $\mathrm{MeV}$ in the simulation, so we simply change only $m_{0}$ to $100 \mathrm{MeV}$ in PNJL calculations done above with $\Lambda=0.6315 \mathrm{GeV}, G_{\mathrm{s}}=5.498$ $\mathrm{GeV}^{-2}, T_{0}=190 \mathrm{MeV}$ and $G_{\mathrm{s} 8}=0$. Figure 12 presents $|\Phi|$ as a function of $T$ in the case of $\theta /(\pi / 3)=0.96$, where the solid (dashed) curve corresponds to the result of PNJL (LQCD). From a qualitative point of view, both the calculations give the same property that the Polykov-loop phase transition is crossover also for this case. From a quantitative point of view, however, LQCD gives a sharper transition than PNJL.

Although more careful and extensive reproduction is necessary in future, now we do the PNJL calculation 

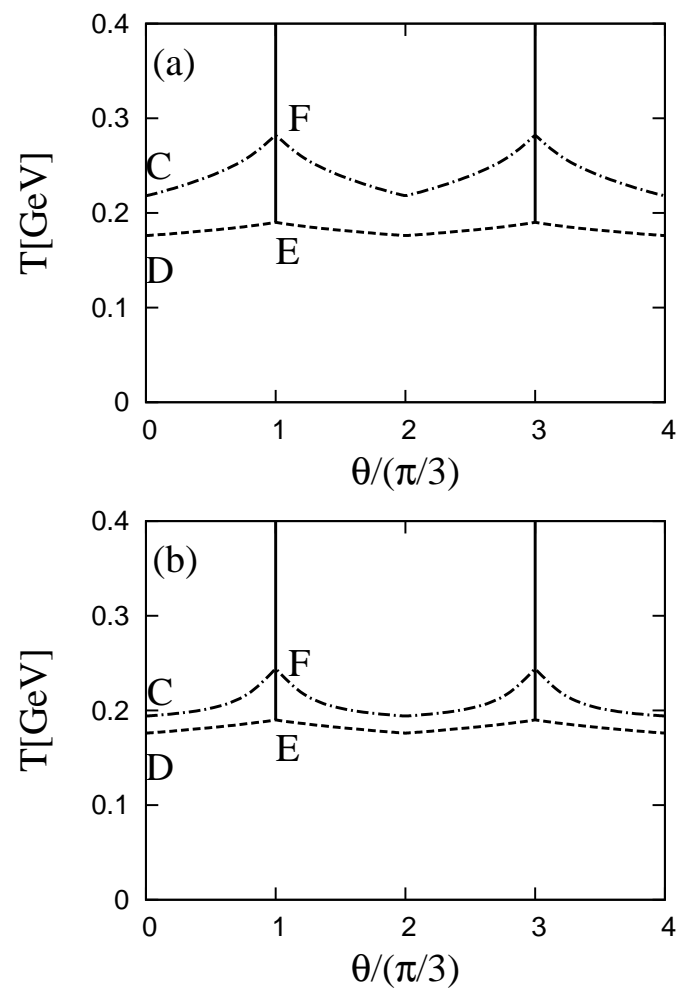

Fig. 11: The phase diagram in the $\theta-T$ plane in the case of $m_{0}=5.5 \mathrm{MeV}$; the eight-quark interaction is switched off in (a) and on in (b). The solid vertical line starting from point $\mathrm{E}$ represents both the Polyakov-loop RW phase transition and the chiral RW phase transition of second order. The dashed curve between $\mathrm{D}$ and $\mathrm{E}$ represents the deconfinement transition of crossover and the dot-dashed curve between $\mathrm{C}$ and $\mathrm{F}$ does the chiral transition of crossover.

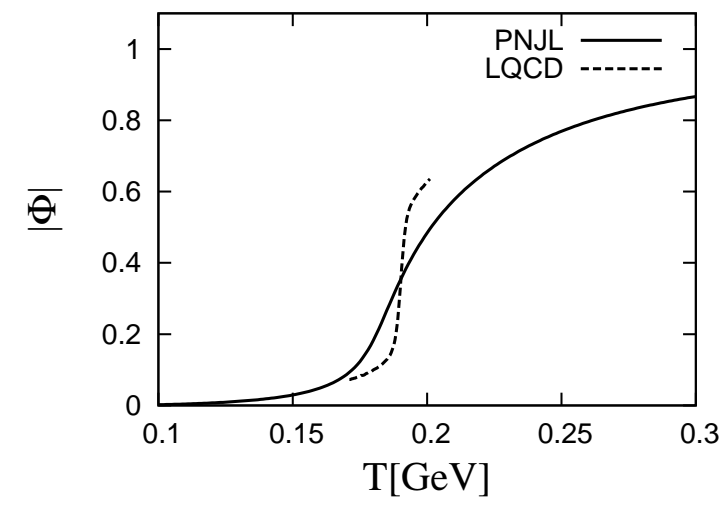

Fig. 12: The absolute value of $\Phi$ as a function of $T$ plane in the case of $\theta /(\pi / 3)=0.96$. The solid curve is the result of PNJL with $m_{0}=100 \mathrm{MeV}$, while the dashed one is the result of lattice QCD [6]. in both the real and imaginary $\mu$ regions with the parameter set determined above. Figure[13(a) presents the chiral phase transition curves in the $\mu^{2}-T$ plane. The solid (dot-dashed) curves represent that the phase transitions are the first-order (crossover), while the dashed curve does the chiral RW phase transition of the secondorder. The curve from point $\mathrm{F}$ to point $\mathrm{A}$ is smooth, as expected. For comparison, in Fig. 13(b) we present the result of $m_{0}=5.5 \mathrm{MeV}$, where the other parameters are fixed. Thus, the phase diagram is largely affected by the change of $m_{0}$. This result encourages us to do more accurate LQCD similations with larger lattice sizes and small current quark mass and also to make more extensive comparison of PNJL calculations with LQCD ones. In the forthcoming paper, we will make such a PNJL extrapolation for the case of four flavor, since most LQCD simulations [4] are done in the case.
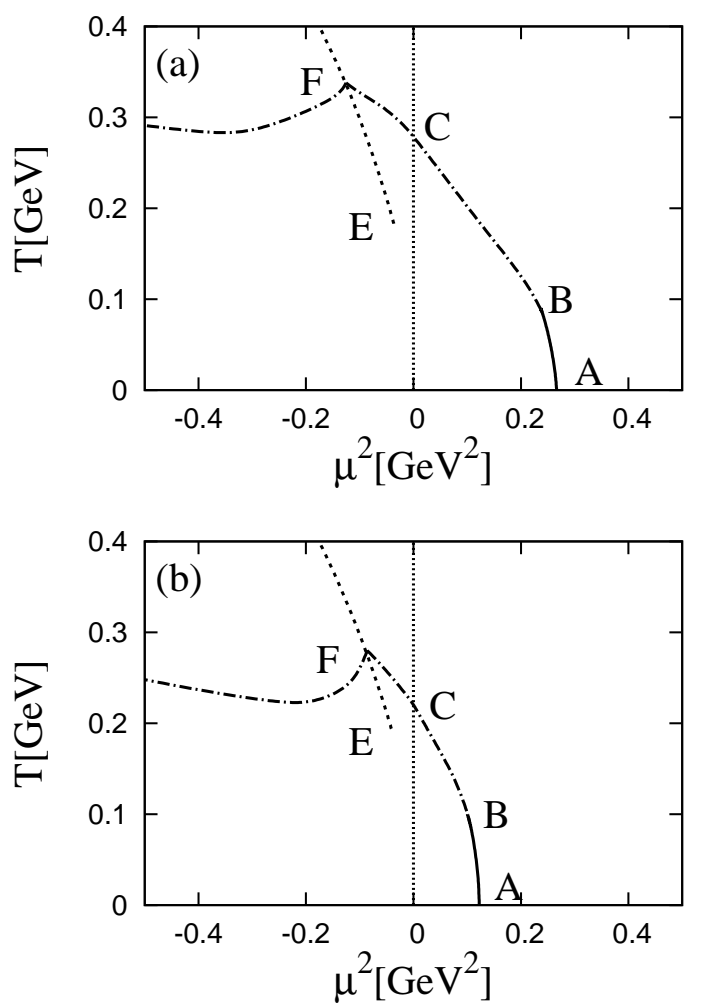

Fig. 13: The phase diagram in the $\mu^{2}-T$ plane for (a) $m_{0}=100 \mathrm{MeV}$ and (b) $m_{0}=5.5 \mathrm{MeV}$. The solid (dotdashed) curves denote the first-order (crossover) chiral phase transitions, while the dashed curve does the chiral RW phase transiton of the second order.

In the lattice simulations [4, 5, 6], the extrapolaton is made in a simple way in which the function

$$
T=\sum_{n=0}^{m} a_{n} \mu^{2 n}
$$

is assumed for the chiral transition curve and the coefficients $a_{n}$ are determined so as to reproduce the lattice 
data in the imaginary $\mu$ region. In this paper the coefficients $a_{n}$ are adjusted to the PNJL result of Fig. 13(b) in the imaginary $\mu$ region. In Fig. 14, four dashed curves labeled (1)-(4) represent results of the simple extrapolation for $m=1,2,3,4$, respectively. The dashed curve of $m=4$ still deviates from the PNJL curve in the real $\mu$ region. Thus, the PNJL curve includes higher-order terms than $m=5$ that the simple extrapolation can not follow accurately. Furthermore, the simple extrapolation can not predict the position of the critical endpoint B. Thus, the PNJL extrapolaiton is superior to the simple extrapolation.

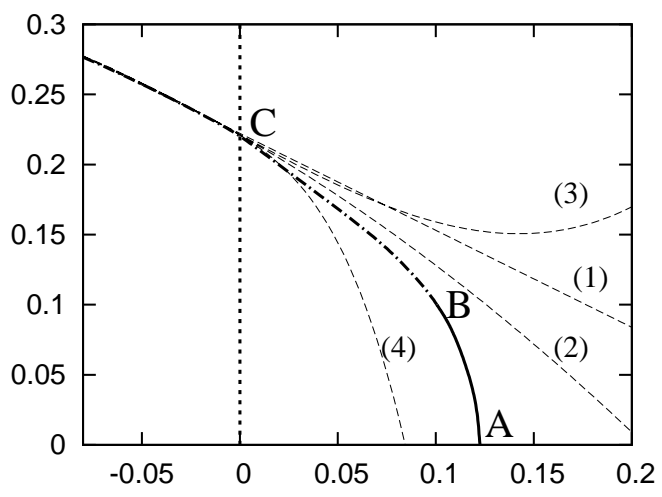

Fig. 14: The phase diagram in the $\mu^{2}-T$ plane based on the extrapolation of (35). Dashed curves labeled (1)-(4) correspond to four cases of $m=1,2,3,4$ in (35), respectively.

\section{SUMMARY}

In summary, we have studied the Polyakov loop extended Nambu-Jona-Lasinio (PNJL) model with imaginary chemical potential. The phase diagram in the $\theta-T$ plane is studied in detail. Since the PNJL model possesses an extended $\mathbb{Z}_{3}$ symmetry, quantities invariant under the symmetry such as the thermodynamic potential, the chiral condensate and the modified Polyakov loop, automatically have the Roberge-Weiss periodicity that QCD does. There appear four types of phase transitions; Polyakov-loop RW transition, chiral RW transition of second order, deconfinement and chiral transitions. The orders of the two RW transitions appearing at $\theta=(2 k+1) \pi / 3$ are determined by the RW periodicity and the even/odd property of the modified Polyakov loop and the chiral condensate. As an interesting feature, the Polyakov-loop RW transition comes out as first order in the imaginary part and the phase of the modified Polyakov loop, but as second order in the real part and the absolute value of the modified Polyakov loop. These results are more informative than the RW prediction [11] and the results of lattice QCD [3, 4, 5, 6, 7].

We have investigated effects of current quark mass $m_{0}$ on the phase diagram. The orders of the ordinary chiral and deconfinement transitions depend on the presence or absence of current quark mass, as expected. In contrast, the orders of the Polyakov-loop RW and the chiral RW transition are not affected by the presence or absence of the current quark mass. The bifurcation point of the chiral transition line, which appears in the phase diagram in the chiral limit, disappears in the case of finite $m_{0}$. We have also studied effects of the scalar-type eight-quark interaction on the phase diagram in the $\theta-T$ plane. It is found that the eight-quark interaction makes the chiral transition temperature shift down to the vicinity of the deconfinement transition temperature in the whole range of $\theta$.

The success of the PNJL model comes from the fact that the PNJL model has the extended $\mathbb{Z}_{3}$ symmetry, more precisely that the thermodynamic potential (18) is a function only of variables, $\Psi, \Psi^{*}, e^{ \pm \beta \mu_{\mathrm{B}}}$ and $\sigma$, invariant under the extended $\mathbb{Z}_{3}$ symmetry. A reliable effective theory of QCD proposed in future is expected to have the same property in its thermodynamic potential. This may be a good guiding principle to elaborate an effective theory of QCD.

Throughout all the present analyses, we can confirm that the results of the present model are consistent with the lattice results. In the analyses of the chiral limit, the present model is even more informative than the lattice simulation. In this paper, we have compared our results with lattice ones only qualitatively, since lattice simulations in the imaginary chemical potential region have been done mainly for the four-flavor case. In the forthcoming paper, we will make quantitative comparison of the present model with lattice QCD. Determining the parameters of our model directly from the lattice results in the imaginary chemical potential region, we will extrapolate the lattice results to the real chemical potential region by using the present model.

\section{Acknowledgments}

The authors thank M. Matsuzaki, H. Fujii, M. Tachibana, K. Miura and T. Murase for useful discussions and suggestions. H.K. also thanks M. Imachi and H. Yoneyama for useful discussions on the RW phase transition. This work has been supported in part by the Grants-in-Aid for Scientific Research (18540280) of Education, Science, Sports, and Culture of Japan.
[1] J. Kogut, M. Stone, H. W. Wyld, W. R. Gibbs, J. Shigemitsu, S. H. Shenker, and D. K. Sinclair, Phys.
Rev. Lett. 50, 393 (1983). 
[2] J. B. Kogut and D. K. Sinclair arXiv:hep-lat/0712.2625 (2007).

[3] P. de Forcrand and O. Philipsen, Nucl. Phys. B642, 290 (2002); P. de Forcrand and O. Philipsen, Nucl. Phys. B673, 170 (2003).

[4] M. D'Elia and M. P. Lombardo, Phys. Rev. D 67, 014505 (2003); M. D'Elia and M. P. Lombardo, Phys. Rev. D 70, 074509 (2004).

[5] H. S. Chen and X. Q. Luo, Phys. Rev. D72, 034504 (2005)

[6] L. K. Wu, X. Q. Luo, and H. S. Chen, Phys. Rev. D76, 034505 (2007).

[7] M. P. Lombardo, arXiv:hep-lat/0612017 (2006).

[8] S. Kratochvila and P. de Forcrand, Prog. Theor. Phys. Suppl. 153, 330 (2004).

[9] P. de Forcrand and S. Kratochvila, Nucl. Phys. B (Proc. Suppl.) 153, 62 (2006).

[10] A. Alexandru, M. Faber, I. Harvaáth, and K. F. Liu, Phys. Rev. D72, 114513-1 (2005).

[11] A. Roberge and N. Weiss, Nucl. Phys. B275, 734 (1986).

[12] Y. Nambu and G. Jona-Lasinio, Phys. Rev. 122, 345 (1961); Phys. Rev. 124, 246 (1961).

[13] P. N. Meisinger, and M. C. Ogilvie, Phys. Lett. B 379, 163 (1996).

[14] A. Dumitru, and R. D. Pisarski, Phys. Rev. D 66, 096003 (2002); A. Dumitru, Y. Hatta, J. Lenaghan, K. Orginos, and R. D. Pisarski, Phys. Rev. D 70, 034511 (2004); A. Dumitru, R. D. Pisarski, and D. Zschiesche, Phys. Rev. D 72, 065008 (2005).

[15] K. Fukushima, Phys. Lett. B 591, 277 (2004).

[16] S. K. Ghosh, T. K. Mukherjee, M. G. Mustafa, and R. Ray, Phys. Rev. D 73, 114007 (2006).

[17] E. Megías, E. R. Arriola, and L. L. Salcedo, Phys. Rev. D 74, 065005 (2006).

[18] C. Ratti, M. A. Thaler, and W. Weise, Phys. Rev. D 73, 014019 (2006).

[19] M. Ciminale, R. Gatto, N. D. Ippolito, G. Nardulli, and M. Ruggieri, Phys. Rev. D 77, 054023 (2008); M. Ciminale, G. Nardulli, M. Ruggieri, and R. Gatto, Phys. Lett. B 657, 64 (2007).

[20] C. Ratti, S. Rößner, M. A. Thaler, and W. Weise, Eur. Phys. J. C 49, 213 (2007).

[21] S. Rößner, C. Ratti, and W. Weise, Phys. Rev. D 75, 034007 (2007).

[22] H. Hansen, W. M. Alberico, A. Beraudo, A. Molinari, M. Nardi, and C. Ratti, Phys. Rev. D 75, 065004 (2007).

[23] C. Sasaki, B. Friman, and K. Redlich, Phys. Rev. D 75, 074013 (2007).

[24] B. J. Schaefer, J. M. Pawlowski, and J. Wambach, Phys.
Rev. D 76, 074023 (2007).

[25] K. Kashiwa, H. Kouno, M. Matsuzaki, and M. Yahiro, Phys. Lett. B 662, 26 (2008).

[26] W. J. Fu, Z. Zhang, and Y. X. Liu, Phys. Rev. D 77, 014006 (2008).

[27] M. Asakawa and K. Yazaki, Nucl. Phys. A504, 668 (1989).

[28] M. Kitazawa, T. Koide, T. Kunihiro, and Y. Nemoto, Prog. Theor. Phys. 108, 929 (2002).

[29] K. Kashiwa, H. Kouno, T. Sakaguchi, M. Matsuzaki, and M. Yahiro, Phys. Lett. B 647, 446 (2007); K. Kashiwa, M. Matsuzaki, H. Kouno, and M. Yahiro, Phys. Lett. B 657, 143 (2007).

[30] Y. Sakai, K. Kashiwa, H. Kouno, and M. Yahiro, Phys. Rev. D 77 051901(R)(2008).

[31] A. A. Osipov, B. Hiller, and J. da Providência, Phys. Lett. B 634, 48 (2006); A. A. Osipov, B. Hiller, J. Moreira, and A. H. Blin, Eur. Phys. J. C 46, 225 (2006); A. A. Osipov, B. Hiller, J. Moreira, A. H. Blin, and J. da Providência, Phys. Lett. B 646, 91 (2007); A. A. Osipov, B. Hiller, J. Moreira, and A. H. Blin, Phys. Lett. B 659, 270 (2008); B. Hiller, A. A. Osipov, A. H. Blin, and J. da Providência, arXiv:hep-ph/0802.3193 (2008).

[32] G. Boyd, J. Engels, F. Karsch, E. Laermann, C. Legeland, M. Lütgemeier, and B. Petersson, Nucl. Phys. B469, 419 (1996).

[33] O. Kaczmarek, F. Karsch, P. Petreczky, and F. Zantow, Phys. Lett. B 543, 41 (2002).

[34] F. Karsch, Lect. notes Phys. 583, 209 (2002).

[35] F. Karsch, E. Laermann, and A. Peikert, Nucl. Phys. B 605, 579 (2002).

[36] M. Cheng et al., Phys. Rev. D 74, 054507 (2006).

[37] S. Weinberg, Physica 96A 327(1979); S. Weinberg, "The quantum theory of fields II", Cambridge University Press (1996).

[38] J. Gasser, and H. Leutwyler, Nucl. Phys. B 250, 465 (1985); J. Gasser, and H. Leutwyler, Phys. Rept. 87, $77(1982)$.

[39] T. A. DeGrand, and C. DeTar, Nucl. Phys. B 225, 590 (1983).

[40] A. Patel, Nucl. Phys. B 243, 411 (1984).

[41] M. Alford, S. Chandrasekharan, J. Cox, and U. -J. Wiese, Nucl. Phys. B 602, 61 (2001).

[42] S. Kim, Ph. de Forcrand, S. Kratochvila, and T. Takaishi, arXiv:hep-lat/0510069 (2005).

[43] P. Hasenfratz, and F. Karsch, Phys. Lett. B 125, 308 (1983). 